\title{
Isotope Dilution LC-ESI-MS/MS and low resolution Selected Reaction Monitoring as a tool for the accurate quantification of urinary testosterone
}

Amaia Ereño Artabe(1), Adriana González-Gago(1), Amanda Suarez Fernández(1), Jorge Pitarch Motellón(2), Antoni F. Roig-Navarro(2), Oscar J. Pozo(3), Pablo Rodríguez-González(1)* and J. Ignacio García Alonso(1)

(1) Department of Physical and Analytical Chemistry. Faculty of Chemistry, University of Oviedo, Spain

(2) Research Institute for Pesticides and Water, University Jaume I, 12071, Castellón, Spain

(3) Integrative Pharmacology and Systems Neuroscience Group, IMIM, Hospital del Mar Medical Research Institute, Doctor Aiguader 88, 08003 Barcelona, Spain

${ }^{*}$ Author for correspondence: rodriguezpablo@uniovi.es, Tel: +34 985103522

\section{Highlights}

- Testosterone quantification in urine by isotope dilution without calibration graphs

- Low resolution Selected Reaction Monitoring to measure isotope distributions

- Minimally ${ }_{13} \mathrm{C}$ labelled testosterone to decrease isotope effects

- Satisfactory accuracy and precision to detect testosterone in different challenging

- scenarios

- Higher sensitivity over standard resolution approaches.

\section{Graphical abstract}
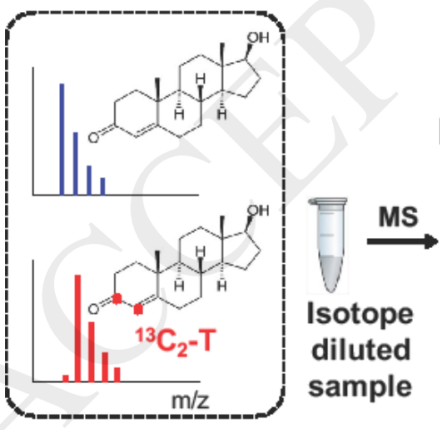

Selected

Reaction

Monitoring

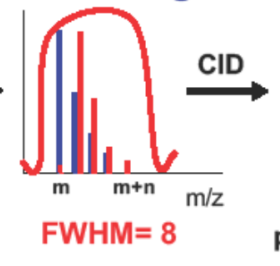

product ions in the sample

\section{ABSTRACT}

A new analytical method for the quantification of testosterone in human urine samples by isotope dilution mass spectrometry is proposed. A standard solution of ${ }^{13} \mathrm{C}_{2}$ testosterone is added to the samples at the beginning of the sample preparation procedure and then the measurements are carried out by UHPLC-ESI-MS/MS. In the 
proposed method, the resolution of the first quadrupole of the tandem MS instrument is reduced to transmit the whole precursor ion cluster to the collision cell and measure the isotopic distribution of the in-cell product ions with a small number of SRM transitions. The construction of a methodological calibration graph is avoided using a labelled analogue previously characterised in terms of concentration and isotopic enrichment in combination with multiple linear regression. In this way, the molar fractions of natural and labelled testosterone are calculated in each sample injection and the amount of endogenous testosterone computed from the known amount of labelled analogue. Recovery values between 97 and $107 \%$ and precisions between 0.4 and $3.7 \%$ (as $\% \mathrm{RSD}$ ) were obtained for testosterone concentrations in urine in the range of 1 to $8 \mathrm{ng}$ $\mathrm{g}^{-1}$. The proposed low resolution SRM methodology was compared for the analysis of human urine samples with the traditional IDMS method based on a calibration graph and the IDMS method based on multiple linear regression combined with standard resolution SRM. A similar accuracy and precision was obtained by the three tested approaches. However, using the low resolution SRM method there was no need to resort to calibration graphs or to specific dedicated software to calculate isotopic distributions by tandem MS and a higher sensitivity was obtained. The proposed low resolution SRM method was successfully applied to the analysis of the certified freezedried human urine NMIA MX005. 


\section{INTRODUCTION}

Testosterone is a natural anabolic androgenic steroid. This hormone is mainly produced by men testicles and women ovaries although a small amount can also be produced by the adrenal glands. It is considered the main masculine hormone but it can also be found in women's blood stream, influencing their sexuality, sexual behaviour, aggressive behaviour and mood [1]. The determination of testosterone is important in health science as it is essential to detect some androgen related disorders, such as infertility in men and women or to evaluate the risk of suffering from certain types of cancer, such as breast [2] or prostate cancer [3]. Additionally, it shows also a great importance in the fields of the doping control and public health as it has been used as doping substance for years, together with some other androgenic anabolic steroids, in high-performance sport and amateurs and gym customers as well [4].

The successful solution of those health-related issues depends on the accurate and precise quantification of steroids. For this reason, analytical strategies improving the quantification figures for steroids are required.

Different analytical methods have been reported for testosterone determination. Some of them deal with the detection of testosterone by immunoassays, including radioimmunoassays (RIA) [5], enzyme immunoassays (ELISA) [6] and immunosensors [7]. These methods are rapid and simple but due to the very similar chemical structures of different steroid hormones and their metabolites they are known to suffer from cross reactivity [5]. Liquid Chromatography coupled to ultraviolet detection (HPLC-UV) has been used for the determination of testosterone, its metabolites and other drugs. Although it provides a higher selectivity than immunoassays the accurate determination of metabolites with similar structure is not possible [8]. Gas Chromatography coupled to Mass Spectrometry (GC-MS) shows higher chromatographic resolution, selectivity and sensitivity than HPLC-UV. Although GC-MS(/MS) is the gold standard technique for steroids determination, testosterone is not a volatile compound so a derivatization stage is required leading to more complex and time consuming sample preparation procedures [9]. Liquid Chromatography coupled to tandem Mass Spectrometry (LCMS/MS) with an electrospray source (ESI) is a very powerful tool for the analysis of hormones and other low molecular weight substances for clinical purposes without requiring any sample derivatisation procedure $[10,11]$. However, a relevant problem with the use of ESI source is the signal variabilities due to matrix effects, which significantly affect the instrumental sensitivity and the accuracy and precision of the results [12]. The most efficient strategy to overcome matrix effects is standardization 
based on the application of Isotope Dilution Mass Spectrometry (IDMS) through the use of stable isotopically labelled standards [13].

Most of the analytical methods for quantification of organic compounds by IDMS require the construction of a calibration graph with mixtures of increasing amounts of the natural abundance compound and a fixed amount of the labelled analogue [14]. Multiply labelled analogues are preferred to avoid spectral overlap between natural and labelled compounds and obtain linear graphs. However, the occurrence of isotope effects increases with multiply labelled analogues, particularly when using deuterated compounds. The occurrence of isotopic effects becomes particularly important when an ESI source is used after the HPLC separation, as time dependent matrix suppression ionisation may occur [15]. Thus, if the tracer and the analyte show different retention times, the accuracy and the precision of the results may be seriously affected. Nevertheless, this classical IDMS approach has been successfully applied to the determination of testosterone in plasma and serum samples by LC-MS/MS showing very good results in terms of accuracy, precision, limits of detection and robustness [24]. Minimally labelled analogues minimise the occurrence of isotopic effects throughout the analytical procedure. However, the spectral overlap between natural and labelled analogue provides non-linear IDMS calibration graphs.

The quantification of organic compounds by IDMS can also be carried out using isotopic distributions and multiple linear regression [16]. This strategy, also known as Isotope Pattern Deconvolution (IPD), provides the direct determination of the molar fractions of analyte and labelled analogue from the experimental mass spectra of the sample. Knowing the amount of labelled analogue added to the sample, a direct quantification of the analyte is provided without resorting to a calibration graph. It has already been applied for the determination of low molecular weight compounds using both, single or tandem mass analysers coupled to GC or HPLC $[17,18]$ and for the absolute quantification of proteins using minimally labelled peptides [19]. More recently, the capabilities of this strategy for the determination of testosterone by LC-MS/MS have been evaluated in the frame of intra- and inter-laboratory reproducibility assays [20].

The successful application of IDMS and multiple linear regression requires accurate and precise measurements of the isotopic distribution of the sample. In addition, the previous determination of the concentration and the isotopic enrichment of the labelled analogue is required. The factors affecting the accuracy and precision of the isotope distribution measurements of organic compounds have not been completely identified 
[14]. Selected ion monitoring (SIM) mode can be easily applied for that purposes but the accurate determination of trace levels or organic molecules in complex matrices require the use of tandem MS. The determination of accurate isotopic distributions of in-cell product ions by Selected Reaction Monitoring (SRM) is not straightforward as the same precursor ion may contribute to different product ions [21]. Thus, up to $n^{2}$ SRM transitions for $\mathrm{n} \mathrm{m/z}$ must be measured and taken into account to calculate the full isotopic distribution of the in-cell product ion. This procedure can be simplified predicting the abundance of each SRM transition using specific dedicated software [22]. An alternative measurement approach is based in the reduction of the resolution of the first quadrupole of the tandem MS instrument, aiming to transmit the whole precursor ion cluster to the collision cell and directly measure the isotopic distribution of the product ion in the second quadrupole [19].

We present here a new analytical methodology for the quantification of the anabolic androgenic steroid testosterone in human urine as a proof of concept that could be eventually applied in future works to other compounds and matrices. The proposed method is based on the addition to the sample of testosterone labelled in two ${ }^{13} \mathrm{C}$ atoms, enzymatic hydrolysis, liquid-liquid extraction and measurement by LC-MS/MS using a low resolution selected reaction monitoring (SRM) mode. The resolution of the first quadrupole is reduced to transmit the whole parent ion cluster to the collision cell for monitoring accurate isotopic distributions of the in-cell product ions using a limited number of SRM transitions. The proposed measurement procedure working at low resolution is compared in terms of accuracy, precision, sensitivity and spectral interferences with the standard SRM mode which requires the use of dedicated software [22] and with the regular IDMS strategy based on a calibration graph. Applicability of the approach is demonstrated by the measurement of the urine concentration of testosterone in real samples and in the Reference Material NMIA MX005.

\section{EXPERIMENTAL}

\subsection{Reagents and materials}

Testosterone ( $\geq 99 \%$ for HPLC) was obtained from Sigma-Aldrich (St. Louis, MA, USA) and $3,4-{ }^{13} \mathrm{C}_{2}$-testosterone (99\%) was obtained from Cambridge Isotope Laboratories (Andover, MA, USA). All the standard solutions were prepared by weight using methanol (LC-MS CHROMASOLV $\geq 99.9 \%$, Sigma-Aldrich) or a $1: 1$ mixture (v/v) of methanol and water. Mobile phase modifiers ammonium formate ( $\geq 99.0 \%)$ and formic 
acid $(98 \%$ p.a.) were purchased from Sigma-Aldrich. Sodium carbonate $(99.999 \%$ for trace analysis, Sigma-Aldrich) and sodium bicarbonate $(99.5 \%)$ from Merck (Darmstadt, Germany) were used to prepare the solid buffer. Ammonium hydrogen phosphate (98\%, Sigma-Aldrich) and hydrochloric acid (37\% for analysis from Merck) were used to prepare the buffer solution. Tert-butyl methyl ether $(99.9 \%$ CHROMASOLV Plus to HPLC from Sigma-Aldrich) was used for the extraction of the urine samples. $\beta$-glucuronidase from $E$. Coli K12 with an enzyme activity of ca. 80 units per mg protein was purchased from Roche (Indianapolis, IN, USA). Ultra-pure water was obtained from a Milli-Q system (Millipore, Bedford, USA). The certified reference material NMIA MX005 consisting in a freeze-dried human urine with certified values for steroid metabolites was purchased to NMI Australia (North Ryde, NSW, Australia)

\subsection{Instrumentation}

A vortex mixer (FB 15024, Fisher Scientific, Hampton, NH, USA) was used for the homogenization of samples and working solutions. A mechanical shaker (Bioblock KL2, Fisher Scientific) was used to homogenize the solid buffer. The samples were incubated in a digital control hotplate RTC Basic (IKA-Werke, Staufen, Germany) for the enzymatic hydrolysis step. A centrifuge Heraeus Multifuge 3 L-R (Thermo Fischer Scientific) was used for the liquid-liquid extraction of the samples. All standard solutions and the mixtures of natural and labelled testosterone were prepared gravimetrically using an analytical balance model MS205DU (Mettler Toledo, Zurich, Switzerland). A liquid chromatograph Agilent 1290 series (Agilent Technologies, Santa Clara, CA) coupled to a triple quadrupole mass spectrometer Agilent 6460 equipped with an electrospray interface (ESI) with jet stream operating in positive ion mode was employed for the analysis of the samples.

\subsection{Procedures}

\subsubsection{Sample preparation}

The sample preparation procedure has been reported in a previous publication [31]. Briefly a gravimetrically controlled amount (between 0.1 and $0.2 \mathrm{~g}$ ) of a $100 \mathrm{ng} \mathrm{g}^{-1}$ solution of ${ }^{13} \mathrm{C}_{2}$-testosterone was added to $2.5 \mathrm{~mL}$ of urine sample. Then, $1 \mathrm{~mL}$ of phosphate buffer $(1 \mathrm{M}, \mathrm{pH}=7)$ and $30 \mu \mathrm{L}$ of the enzyme $\beta$-glucuronidase $E$. Coli were added, and the blend was incubated for 1 hour at $55^{\circ} \mathrm{C}$. Once the samples reached room temperature, $200 \mathrm{mg}$ of solid buffer were added and dissolved by manual 
shaking. Liquid-liquid extraction of the sample was carried out adding $6 \mathrm{~mL}$ of methyl tert-butyl ether and stirring in a vortex mixer for 1 minute. The mixture was centrifuged for $5 \mathrm{~min}$ at $3500 \mathrm{rpm}$ and the organic layer transferred to a new vial, evaporated until dryness and redissolved in $300 \mu \mathrm{L} \mathrm{MeOH/H} / \mathrm{H}_{2} \mathrm{O}(1: 1, \mathrm{v} / \mathrm{v})$. Finally, $5 \mu \mathrm{L}$ of the extract were injected in the LC-ESI-MS/MS system.

The freeze-dried urine certified reference material was prepared following the reconstitution protocol described in its certificate of analysis. Briefly, after reaching room temperature, $20 \mathrm{~g}$ of ultrapure water were added gravimetrically to the freezedried material. After a gentle manual shaking of the vial to dissolve all the solid material and rinse the container walls, the reconstituted material was heated for $30 \mathrm{~min}$ at 40 ${ }^{\circ} \mathrm{C}$. After equilibration to room temperature, $2 \mathrm{~mL}$ aliquots were taken and treated according to the sample preparation procedure described above.

\subsubsection{Measurements by HPLC-ESI-MS/MS}

The analysis of the samples was carried out using an Agilent Zorbax SB-C18 column $(2.1 \mathrm{~mm} \times 50 \mathrm{~mm}, 1.8 \mu \mathrm{m})$ at $0.3 \mathrm{~mL} \mathrm{~min}^{-1}$ flow rate. $5 \mu \mathrm{L}$ was selected as injection volume for both standards and sample extracts. Mobile phases $A$ and $B$ were water and methanol, both with $0.1 \%$ formic acid and $1 \mathrm{mM}$ ammonium formate. The chromatographic conditions were adapted from the method proposed elsewhere [23]. Briefly, a gradient starting with $45 \%$ B for $1 \mathrm{~min}$, from 45 to $50.3 \%$ of B until 9 min, from 50.3 to $95 \%$ B until $9.5 \mathrm{~min}, 95 \%$ B until $10.5 \mathrm{~min}$, from 95 to 45 within $0.5 \mathrm{~min}$ and $45 \%$ B for 3 min was applied. The ionization source working conditions were $5000 \mathrm{~V}$ as capillary voltage, $45 \mathrm{psi}$ as nebuliser pressure, $11 \mathrm{~L} \mathrm{~min}^{-1}$ as drying gas flow rate and $320{ }^{\circ} \mathrm{C}$ as drying gas temperature. The sheath gas flow rate and temperature were 12 $\mathrm{L} \mathrm{min}^{-1}$ and $200{ }^{\circ} \mathrm{C}$, respectively. The fragmentor voltage applied was $100 \mathrm{~V}$ and the collision energy was $20 \mathrm{eV}$.

When the samples were measured in SIM mode the $\mathrm{m} / \mathrm{z}$ values $289.1,290.1,291.1$ and 292.1 were acquired. When the samples were measured using the standard SRM mode at conventional unit resolution in the first quadrupole the selected operating transitions were $289.1 \rightarrow 97.1,290.1 \rightarrow 98.1,291.1 \rightarrow 99.1,292.1 \rightarrow 99.1$ for the fragment ion at $\mathrm{m} / \mathrm{z} 97$ and $289.1 \rightarrow 109.1,290.1 \rightarrow 110.1,291.1 \rightarrow 111.1,292.1 \rightarrow 111.1$ for the fragment ion at $\mathrm{m} / \mathrm{z} 109$.

When the samples were measured using the low resolution SRM mode the mass resolution of the first quadrupole was modified to a Full Width at Half Maximum 
(FWHM) value of $8 \mathrm{u}$. For the fragment ion at $\mathrm{m} / \mathrm{z} 109$ the following SRM transitions were measured: $289.1 \rightarrow 109.1,289.1 \rightarrow 110.1,289.1 \rightarrow 111.1,289.1 \rightarrow 112.1$. For the fragment ion at $\mathrm{m} / \mathrm{z} 97$, the measured SRM transitions were $289.1 \rightarrow 97.1,289.1 \rightarrow 98.1$, $289.1 \rightarrow 99.1,289.1 \rightarrow 100.1$.

For the traditional IDMS procedure based on a gravimetrically corrected calibration graph the SRM transitions acquired were $289.1 \rightarrow 97.1$ and $291.1 \rightarrow 99.1$ for the fragment ion at $\mathrm{m} / \mathrm{z} 97$ and $289.1 \rightarrow 109.1$ and $291.1 \rightarrow 111.1$ for the fragment ion $\mathrm{at} \mathrm{m} / \mathrm{z}$ 109.

\subsubsection{Calculation of testosterone concentrations by IDMS and multiple linear regression}

When applying IDMS and multiple linear regression with tandem MS the measured isotopic distribution (from $\mathrm{i}=1$ to $\mathrm{i}=\mathrm{n}$ isotopologues) of a given cell product ion in the sample $A_{\text {mixture }}$, can be assumed to be a linear combination of the isotope distribution of natural abundance product ion, $A_{\text {natural, }}$ and that of the isotopically labelled product ion, $A_{\text {tracer. }}$ The relative contribution of both isotope patterns in the experimental mass spectrum are the molar fractions $x_{\text {natural }}$ and $x_{\text {tracer }}$ which can be calculated by solving equation (1):

$$
\left[\begin{array}{c}
A_{\text {mixture }}^{1} \\
\vdots \\
A_{\text {mixture }}^{n}
\end{array}\right]=\left[\begin{array}{cc}
A_{\text {natural }}^{1} & A_{\text {tracer }}^{1} \\
\vdots & \vdots \\
A_{\text {natural }}^{n} & A_{\text {tracer }}^{n}
\end{array}\right] \cdot\left[\begin{array}{c}
x_{\text {natural }} \\
x_{\text {tracer }}
\end{array}\right]+\left[\begin{array}{c}
e^{1} \\
\vdots \\
e^{n}
\end{array}\right]
$$

To apply this strategy the isotope patterns of the natural and labelled testosterone product ions must be known in advance. Since the molar fractions are the only unknowns in Equation 1 an error vector must be included in the equation in such a way that the molar fractions can be calculated by multiple linear regression. Knowing the molar fractions of natural and labelled testosterone ( $x_{\text {natural }}$ and $x_{\text {tracer }}$, respectively) and the amount (moles) of labelled analogue $\left(\mathrm{N}_{\text {tracer }}\right)$ initially added to the sample, the amount (moles) of testosterone in the sample $\left(\mathrm{N}_{\text {natural }}\right)$ can be obtained by applying equation (2).

$$
\frac{N_{\text {natural }}}{N_{\text {tracer }}}=\frac{x_{\text {natural }}}{x_{\text {tracer }}}
$$




\section{RESULTS AND DISCUSSION}

\subsection{Measurement of the isotope composition of testosterone by HPLC-ESI- MS/MS}

First, the fragmentation pattern of testosterone in the LC-ESI-MS/MS system was studied measuring standards of natural abundance or isotopically labelled testosterone in the product ion scan mode. The obtained mass spectrum shown in Figure 1 is in agreement with previously reported fragmentation of testosterone by Collision Induced Dissociation (CID) [24-26]. The two main product ions (at $m / z=97$ and $m / z=109$ ) obtained when selecting the precursor ion at $\mathrm{m} / \mathrm{z}=289$ were used for the development of the analytical methods.

The application of IDMS with multiple linear regression requires the knowledge of the isotopic distribution of the analyte and the labelled analogue as well as the accurate and precise measurement of the isotopic composition of the isotope-diluted sample. When working with single MS, the isotopic distribution of the natural and labelled analogue can be either experimentally measured or calculated by standard software based on polynomial distributions taking into account the isotopic abundance of the constituting elements [27-29]. However, when working with tandem MS the real isotopic distribution of an in-cell fragment ion of $n$ isotopologues obtained after collision induced dissociation (CID) cannot be directly measured only by $n$ SRM transitions. The reason behind this is that several isotopologues of the precursor molecule may contribute to the same isotopologue of the in-cell fragment ion. Therefore, the measurement of the full isotopic distribution of an in-cell molecular fragment ion requires a high number of transitions (up to $\mathrm{n}^{2}$ ).

Alternatively, the isotope distributions of in-cell fragment ions measured by SRM can be theoretically calculated knowing the fragmentation mechanism or predicted by suitable SRM dedicated software such as IsoPatrn@ [22]. This allows the application of IDMS with multiple linear regression measuring a limited number of SRM transitions. In addition, we have recently developed in our laboratory an alternative method based on the reduction of the mass resolution of the first quadrupole to transmit the whole precursor ion cluster to the collision cell [19]. In this way, the full isotopic distribution of an in-cell fragment ion can be directly measured with a small number of SRM transitions. Thus, the obtained isotopic distribution can be directly compared with those calculated by standard software based on polynomial distributions of the isotope abundances of the different elements constituting the in-cell fragment ion $[27,28]$. Low resolution SRM can be applied in combination with minimally labelled analogues as the 
same mass window in the first quadrupole can be used to transmit both the natural abundance and the labelled analogue. Minimally labelled analogues are less prone to isotope effects than multiply labelled analogues (especially multiply deuterated compounds).

The quantification by IDMS using the low resolution SRM method requires the transmission of at least two isotopologues for each analogue (natural and labelled). Therefore, the resolution of the first quadrupole can be optimised to ensure a complete transmission while minimising the risk of spectral interferences when analysing real samples. A mixture of natural and ${ }^{13} \mathrm{C}_{2}$-testosterone was measured at different resolutions of the first quadrupole. To do so, the slope and intercept of the scan line were modified to obtain different FWHM values. Precursor ion scans from $\mathrm{m} / \mathrm{z} 278$ to 296 of a mixture of natural abundance and ${ }^{13} \mathrm{C}_{2}$-testosterone for product ions from $\mathrm{m} / \mathrm{z}$ 97 to 100 and from 109 to 112 were acquired at three different values of FWHM (7.9, 9.2, and $11.6 \mathrm{u}$ ). The obtained spectra are shown in Figure 2. As can be observed, when working at $\mathrm{FWHM}=11.6$ the intensities of the all product ions are constant over $a$ precursor ion range from $\mathrm{m} / \mathrm{z} 284$ to 290 . When working at $F W H M=9.2$ the intensities of the all product ions are constant over a precursor ion range of $\mathrm{m} / \mathrm{z}=286-290$ and when working at $\mathrm{FWHM}=7.9$ the intensities of the all product ions are constant over a precursor ion range of $\mathrm{m} / \mathrm{z}=288-290$. According to these results the three resolutions would provide a complete transmission of the four precursor ions to the collision cell by setting a $\mathrm{m} / \mathrm{z}$ value of 289 in the first quadrupole.

\subsection{Determination of the isotopic enrichment of the ${ }^{13} C_{2}$-testosterone}

The application of IDMS using multiple linear regression requires the accurate characterisation of the labelled analogue in terms of isotopic enrichment and concentration. The isotopic enrichment of ${ }^{13} \mathrm{C}_{2}$ testosterone can be obtained from the measurement of its experimental isotopic distributions following the procedure described elsewhere [29]. This procedure takes into account the limited resolution of quadrupoles by which the signal measured for each $\mathrm{m} / \mathrm{z}$ may be affected by peak tailing from adjacent $\mathrm{m} / \mathrm{z}$. A standard solution of ${ }^{13} \mathrm{C}_{2}$-testosterone was injected into the LC-MS/MS and the isotopic distribution of the precursor ion at 291 was measured in SIM mode. Also, the isotopic distribution of the in-cell fragment ions at $\mathrm{m} / \mathrm{z} 99$ and 111 were measured at a resolution of $\mathrm{FWHM}=11.6$ to transmit the whole cluster for the precursor ion. Table 1 shows the isotopic enrichment values obtained in SIM and in low resolution SRM for both product ions for three different aliquots of the ${ }^{13} \mathrm{C}_{2}$-testosterone 
measured in three different days. As can be observed the average values of the isotopic enrichment obtained by SIM and low resolution SRM were in agreement. Finally, an average of the three different values $99.27 \pm 0.12$ was taken as reference value.

\subsection{Determination of the concentration of the ${ }^{13} \mathrm{C}_{2}$-testosterone}

The concentration of the ${ }^{13} \mathrm{C}_{2}$-testosterone solution was quantified by reverse IDMS. In order to select the optimal mass resolution, the measurements were made using the three different FWHM values (7.9, 9.2, and 11.6) evaluated before. Three independent mixtures of a natural abundance testosterone standard and the labelled testosterone were prepared and injected in triplicate in the HPLC-ESI-MS/MS system. Both product ions (at $\mathrm{m} / \mathrm{z}=97$ and 109) were used for quantitation in order to check for potential interfering ions that might be transferred to the second quadrupole. The average concentration values obtained from each resolution and for each product ion are summarised in Table 2. The results are compared with the concentration obtained by SIM at standard resolution using the isotopic distribution of the precursor ion $(\mathrm{m} / \mathrm{z}$ values 289.1, 290.1, 291.1 and 292.1). As can be observed in Table 2 the concentration values obtained by SIM measuring the precursor ion cluster at $\mathrm{m} / \mathrm{z} 289.1$ were in agreement with those obtained by low resolution SRM for both in-cell fragment ions and the three different FWHM values. A FWHM value of 7.9 was finally selected to minimise the transmission of potential interfering ions that might be present in real samples. A central mass of 289 was selected as precursor ion for the development of the quantification method in agreement with the results obtained in section 3.1 .

\subsection{Calculation of the limit of detection and limit of quantification of the proposed low resolution SRM method}

The limit of detection (LOD) and limit of quantification (LOQ) of the proposed method were estimated calculating the blank values obtained in ultrapure water for the two product ion clusters. Six independent replicates of ultrapure water were spiked with the labelled analogue and analysed as described in the sample preparation procedure. The standard deviation (SD) of the obtained blank values was used to calculate the LOD (3SD) and LOQ (10SD). The obtained LOD was $0.007 \mathrm{ng} \mathrm{g}^{-1}$ and the LOQ was 0.024 $\mathrm{ng} \mathrm{g}^{-1}$ for the two product ion clusters (Table 3) which are well below the expected concentration levels of testosterone in real urine samples. A more reliable LOD and 
LOQ of the method could not be measured for urine samples as it was not possible to obtain a testosterone-free actual urine matrix.

\subsection{Recovery studies in fortified ultrapure water samples}

Recovery studies using the methodology based on low resolution SRM were carried out first with fortified ultrapure water samples using the two product ion clusters selected for the quantification (at $m / z=97$ and $m / z=109$ ). Increasing amounts of natural abundance testosterone were added to ultrapure water to obtain fortified samples at a concentration level of $0.25,0.75,4$ and $7.5 \mathrm{ng} \mathrm{g}^{-1}$. Then, a similar amount of labelled testosterone was added to each sample. Two or three independent replicates were prepared for each concentration level and each replicate were injected in triplicate in the LC-ESI-MS/MS system. Recovery values were calculated for each replicate and the results obtained are shown in Table 4. As can be observed, if we exclude the values obtained for the product ion cluster at $\mathrm{m} / \mathrm{z} 97$ at $0.25 \mathrm{ppb}(88.3 \pm 1.7 \%)$, the recoveries obtained for all levels and clusters were between 98 and 103\%. The precision of the concentration values expressed as RSD was between 0.1 and $4.7 \%$. For concentration levels higher than $0.25 \mathrm{ng} \mathrm{g}^{-1}$, the same level of accuracy and precision was obtained for the two product ion clusters indicating the absence of any interfering substance, as expected in the absence of matrix.

\subsection{Recovery studies in urine samples}

Recovery studies using the low resolution SRM methodology were carried out in a fortified human urine sample. The sample was previously analysed ( $n=3$ independent replicates) following the sample preparation procedure described in the experimental section obtaining an average concentration of $1.095 \pm 0.016 \mathrm{ng} \mathrm{g}^{-1}$ for the product ion cluster at $\mathrm{m} / \mathrm{z}=97$ and $1.043 \pm 0.016$ for the product ion cluster at $\mathrm{m} / \mathrm{z}=109$. Then, the sample was fortified with natural abundance testosterone to four different concentration levels $\left(1.25,1.7,4.7\right.$ and $\left.8 \mathrm{ng} \mathrm{g}^{-1}\right)$ and analysed following the procedure described in section 2.3.1. Three independent replicates were prepared for each concentration level and each replicate was injected in triplicate in the LC-ESI-MS/MS system. Recovery values were calculated for each replicate and the results obtained are shown in Table

5. As can be observed, the recoveries obtained for all levels and clusters were between 97 and $107 \%$. The precision of the concentration values expressed as RSD was between 0.4 and $3.7 \%$. 


\subsection{Comparison of different methods for the determination of testosterone in urine samples}

The proposed low resolution SRM methodology was compared with the traditional IDMS method based on a calibration curve and the IDMS method based on multiple linear regression combined with standard resolution SRM. For that purpose, five different samples from two men and three women were collected and analysed following the procedure described in section 2.3.1. Considering the typical levels of testosterone in urine samples from men and women, the amount of spike added to the male urines was twice higher than that added to female urines. Then, all the samples were treated following the sample preparation procedure described in the experimental section and the sample extracts were measured in triplicate by the three quantification methods. The method based on a calibration graph required the previous injection of standard solutions at different concentration levels whereas the methods based on multiple linear regression provided a concentration of the samples from each sample injection.

The results obtained for the urine samples are summarised in Table 6. The three methods provided very similar concentration values for all samples except for the most concentrated (M1) and for the less concentrated sample (F3). For sample M1 the method based on standard resolution SRM provided a significantly higher value than the other methods whereas for sample F3 the low resolution SRM method provided a significantly lower (first transition) or higher (second transition) value than the other methods. The precision achieved by these methods after the three replicate injections is comparable. The relative standard deviation range obtained for the samples was from 0.1 to $2.6 \%$ for the low resolution SRM method, from 0.1 to $2.1 \%$ for the standard resolution SRM and from 0.1 to $8.5 \%$ for the classical IDMS method based on a calibration graph.

According to these results, the three methods can be considered equivalent and suitable for the reliable determination of testosterone in urine samples but the low resolution SRM method provides several advantages over the other methods. First, when comparing the low resolution SRM with the classical IDMS method, the preparation and measurement of a calibration graph is not required for quantification. Therefore, the total analysis time and the consumption of labelled material are significantly lower. Secondly, if the low resolution SRM method is compared with the standard resolution SRM method, the advantage relies on the direct measurement of 
isotope abundances of the analyte and the tracer without the need of resorting to any specific-dedicated software [30]. Moreover, an increased ion transmission to the collision cell is achieved leading to a significantly increase in the method sensitivity compared to the standard resolution SRM method. This effect can be observed in Figure 3, which shows a LC-MS/MS chromatogram of the urine sample of $1.5 \mathrm{ng} \mathrm{g}^{-1}$ measured with standard and low resolution SRM. According to these results, the proposed method can be a good alternative for the determination of testosterone in those cases in which low concentration levels need to be detected such as hypogonadism diagnosis through testosterone determination in human serum [30] or to decrease the urine sample size to avoid matrix effects.

\subsection{Analysis of NMIA MX005 reference material}

The low resolution SRM method was finally applied to the analysis of the certified reference material NMIA MX005 consisting in a freeze-dried human urine with certified values for steroid metabolites. Nine independent replicate samples were prepared as described in the experimental section in three different working days and each replicate was injected in triplicate in the LC-MSMS system. As can be observed in Table 7 the results obtained were in good agreement with the certified values. Very good precision was attained. The relative standard deviation obtained from the analysis of nine replicates was lower than $1.7 \%$ RSD.

\section{CONCLUSIONS}

The results obtained in this work demonstrates that the proposed IDMS method based on multiple linear regression and low resolution SRM provides accurate and precise determinations of testosterone in human urine samples. The method shows several advantages over previously published procedures. When compared with the classical IDMS, the preparation and measurement of a calibration graph is not required for quantification and hence, the total analysis time and the consumption of labelled material are significantly reduced. When comparing the low resolution SRM with the standard resolution SRM method, the isotope abundances of the analyte and the tracer can be easily obtained from accurate experimental measurements instead of resorting to specific-dedicated software [30]. In addition, the low resolution SRM method provides a significant increase of the sensitivity enabling the quantification of low testosterone concentrations in urine samples. The excellent precision attained by the 
developed method shows its great potential for the accurate determination of testosterone in biological matrices. The application of this strategy would provide the sensitivity and accuracy required for different challenging scenarios such as (i) to quantify of the low amounts of testosterone in post-menopausal women, (ii) to test the efficacy of androgen deprivation therapies or (iii) to control individual limits of testosterone or other endogenous steroids in doping control.

\section{ACKNOWLEDGEMENTS}

The authors are grateful for financial support from the Spanish Ministry of Economy and Competitiveness through Project Ref. CTQ2015-70366-P, co-funded by FEDER. Adriana González Gago acknowledges the financial support received from the ClarínMarie Curie Cofound program (ACB 14-09). Spanish Health National System is acknowledged for Oscar J. Pozo contract (CPII16/00027).

\section{REFERENCES}

[1] K. Christiansen, Behavioural correlates of testosterone, in: Testosterone, 1998: pp. 107-142. doi:10.1017/cbo9780511545221.005.

[2] N.C. Onland-Moret, R. Kaaks, P. a H. van Noord, S. Rinaldi, T. Key, D.E. Grobbee, P.H.M. Peeters, Urinary endogenous sex hormone levels and the risk of postmenopausal breast cancer., Br. J. Cancer. 88 (2003) 1394-1399. doi:10.1038/sj.bjc.6600890.

[3] A. Pichon, Y. Neuzillet, H. Botto, J.-P. Raynaud, C. Radulescu, V. Molinié, J.-M. Herve, T. Lebret, Preoperative low serum testosterone is associated with high grade prostate cancer and an increased Gleason score upgrading, Prostate Cancer P. D. 387 (2015) 1-6. doi:10.1038/pcan.2015.44.

[4] F. Sjöqvist, M. Garle, A. Rane, Use of doping agents, particularly anabolic steroids, in sports and society, Lancet. 371 (2008) 1872-1882. doi:10.1016/S0140-6736(08)60801-6.

[5] V. Moal, E. Mathieu, P. Reynier, Y. Malthièry, Y. Gallois, Low serum testosterone assayed by liquid chromatography-tandem mass spectrometry. Comparison with five immunoassay techniques, Clin. Chim. Acta. 386 (2007) 12-19. doi:10.1016/j.cca.2007.07.013.

[6] L.T. Di Benedetto, T. Dimitrakopoulos, R.M. Davy, P.J. Iles, Testosterone Determination Using Rapid Heterogeneous Competitive-Binding for EnzymeLinked Immunosorbent Assay in Flow Injection., Anal. Lett. 29 (1996) 21252139. doi:10.1080/00032719608002236.

[7] G. Conneely, M. Aherne, H. Lu, G.G. Guilbault, Development of an immunosensor for the detection of testosterone in bovine urine, Anal. Chim. Acta. 583 (2007) 153-160. doi:10.1016/j.aca.2006.09.062.

[8] G. Wang, H. Xiaoming Cui, K.-C. Cheng, W.A. Korfmacher, Ultra-performance 
liquid chromatography/tandem mass spectrometric determination of testosterone and its metaolites in vitro samples, Rapid Commun. Mass Spectrom. 20 (2006) 2215-2221. doi:10.1002/rcm.

[9] P. Caron, V. Turcotte, C. Guillemette, A chromatography/tandem mass spectrometry method for the simultaneous profiling of ten endogenous steroids, including progesterone, adrenal precursors, androgens and estrogens, using low serum volume, Steroids. 104 (2015) 16-24. doi:10.1016/j.steroids.2015.07.009.

[10] M.M. Kushnir, A.L. Rockwood, W.L. Roberts, B. Yue, J. Bergquist, A.W. Meikle, Liquid chromatography tandem mass spectrometry for analysis of steroids in clinical laboratories, Clin. Biochem. 44 (2011) 77-88.

doi:10.1016/j.clinbiochem.2010.07.008.

[11] J. Marcos, O.J. Pozo, Current LC-MS methods and procedures applied to the identification of new steroid metabolites, J. Steroid Biochem. Mol. Biol. 162 (2016) 41-56. doi:10.1016/j.jsbmb.2015.12.012.

[12] A. Furey, M. Moriarty, V. Bane, B. Kinsella, M. Lehane, Ion suppression; A critical review on causes, evaluation, prevention and applications, Talanta. 115 (2013) 104-122. doi:10.1016/j.talanta.2013.03.048.

[13] A. González-Antuña, J.C. Domínguez-Romero, J.F. García-Reyes, P. Rodríguez-González, G. Centineo, J.I. García Alonso, A. Molina-Díaz, Overcoming matrix effects in electrospray: Quantitation of $\beta$-agonists in complex matrices by isotope dilution liquid chromatography-mass spectrometry using singly13C-labeled analogues, J. Chromatogr. A. 1288 (2013) 40-47. doi:10.1016/j.chroma.2013.02.074.

[14] J.I. Garcia Alonso, P. Rodriguez-Gonzalez, Isotope Dilution Mass Spectrometry, Royal Society of Chemistry, Cambridge (UK), 2013.

[15] J. Wieling, LC-MS-MS experiences with internal standards, Chromatographia. 55 (2002) S107-S113. doi:10.1007/BF02493365.

[16] A. González-Antuña, P. Rodríguez-González, G. Centineo, J.I. García Alonso, Evaluation of minimal 13C-labelling for stable isotope dilution in organic analysis., Analyst. 135 (2010) 953-964. doi:10.1039/b924432h.

[17] A. González-Antuña, P. Rodríguez-González, I. Lavandera, G. Centineo, V. Gotor, J.I.G. Alonso, Development of a routine method for the simultaneous confirmation and determination of clenbuterol in urine by minimal labeling isotope pattern deconvolution and GC-El-MS, Anal. Bioanal. Chem. 402 (2012) 1879-1888. doi:10.1007/s00216-011-5611-1.

[18] M. Fernández-Fernández, P. Rodríguez-González, M.E. Añón Álvarez, F. Rodríguez, F. V. Álvarez Menéndez, J.I. García Alonso, Simultaneous Determination of Creatinine and Creatine in Human Serum by Double-Spike Isotope Dilution Liquid Chromatography-Tandem Mass Spectrometry (LCMS/MS) and Gas Chromatography-Mass Spectrometry (GC-MS), Anal. Chem. 87 (2015) 3755-3763. doi:10.1021/acs.analchem.5b00769.

[19] A. González-Antuña, P. Rodríguez-González, R. Ohlendorf, A. Henrion, V. Delatour, J.I. García Alonso, Determination of Cystatin C in human serum by isotope dilution mass spectrometry using mass overlapping peptides, J. Proteomics. 112 (2014) 141-155. doi:10.1016/j.jprot.2014.09.005.

[20] J. Pitarch-Motellón, A.F. Roig-Navarro, J.V. Sancho, M. Ibáñez, N. FabregatCabello, O.J. Pozo, R. Ventura, J.I.G. Alonso, P. Rodríguez-González, A.G. 
Gago, A.E. Artabe, P. Van Eenoo, K. Deventer, Y. Dehnes, S. Rzeppa, Evaluation of uncertainty sources in the determination of testosterone in urine by calibration-based and isotope dilution quantification using ultra high performance liquid chromatography tandem mass spectrometry, J. Chromatogr. A. 1508 (2017) 73-80. doi:10.1016/j.chroma.2017.05.072.

[21] Á. Castillo, E. Gracia-Lor, A.F. Roig-Navarro, J.V. Sancho, P. RodríguezGonzález, J.I.G. Alonso, Isotope pattern deconvolution-tandem mass spectrometry for the determination and confirmation of diclofenac in wastewaters, Anal. Chim. Acta. 765 (2013) 77-85. doi:10.1016/j.aca.2012.11.033.

[22] L. Ramaley, L. Cubero Herrera, Software for the calculation of isotope patterns in tandem mass spctrometry, Rapid Commun. Mass Spectrom. 22 (2008) 27072714. doi:10.1002/rcm.

[23] A. Fabregat, O.J. Pozo, J. Marcos, J. Segura, R. Ventura, Quantification of testosterone and metabolites released after alkaline treatment in human urine, Drug Test. Anal. 2 (2010) 630-636. doi:10.1002/dta.227.

[24] T.M. Williams, A.J. Kind, E. Houghton, D.W. Hill, Electrospray collision-induced dissociation of testosterone and testosterone hydroxy analogs, J. Mass Spectrom. 34 (1999) 206-216. doi:10.1002/(SICI)10969888(199903)34:3<206::AID-JMS785>3.0.CO;2-1.

[25] O.J. Pozo, P. Van Eenoo, K. Deventer, S. Grimalt, J. V. Sancho, F. Hernandez, F.T. Delbeke, Collision-induced dissociation of 3-keto anabolic steroids and related compounds after electrospray ionization. Considerations for structural elucidation, Rapid Commun. Mass Spectrom. 22 (2008) 4009-4024. doi:10.1002/rcm.

[26] M. Thevis, S. Beuck, S. Höppner, A. Thomas, J. Held, M. Schäfer, J. Oomens, W. Schänzer, Structure elucidation of the diagnostic product ion at $\mathrm{m} / \mathrm{z} 97$ derived from androst-4-en-3-one- based steroids by ESI-CID and IRMPD spectroscopy, J. Am. Soc. Mass Spectrom. 23 (2012) 537-546. doi:10.1007/s13361-011-0308-4.

[27] H. Kubinyi, Calculation of isotope distributions in mass spectrometry. A trivial solution for a non-trivial problem, Anal. Chim. Acta. 247 (1991) 107-119. doi:10.1016/S0003-2670(00)83059-7.

[28] J.I. García Alonso, P. Rodríguez-González, A. González-Gago, A. GonzálezAntuña, Determination of the uncertainties in the theoretical mass isotopomer distribution of molecules, Anal. Chim. Acta. 664 (2010) 68-76. doi:10.1016/j.aca.2010.01.063.

[29] A. González-Antuña, P. Rodríguez-González, J.I. García Alonso, Determination of the enrichment of isotopically labelled molecules by mass spectrometry, J. Mass Spectrom. 49 (2014) 681-691. doi:10.1002/jms.3397.

[30] M. Livingston, A. Kalansooriya, A.J. Hartland, S. Ramachandran, A. Heald, Serum testosterone levels in male hypogonadism: Why and when to check $-A$ review, (2017) 1-9. doi:10.1111/ijcp.12995. 


\section{FIGURES}

Figure 1. Product ion scan for the precursor ion at $\mathrm{m} / \mathrm{z} 289.1$ obtained by LC-ESI-MSMS. The chemical structures of the precursor ion and the two main product ions at $\mathrm{m} / \mathrm{z} 97.0$ and 109.0 are given. The structure of the fragment ions is given according to previous works [25,26].

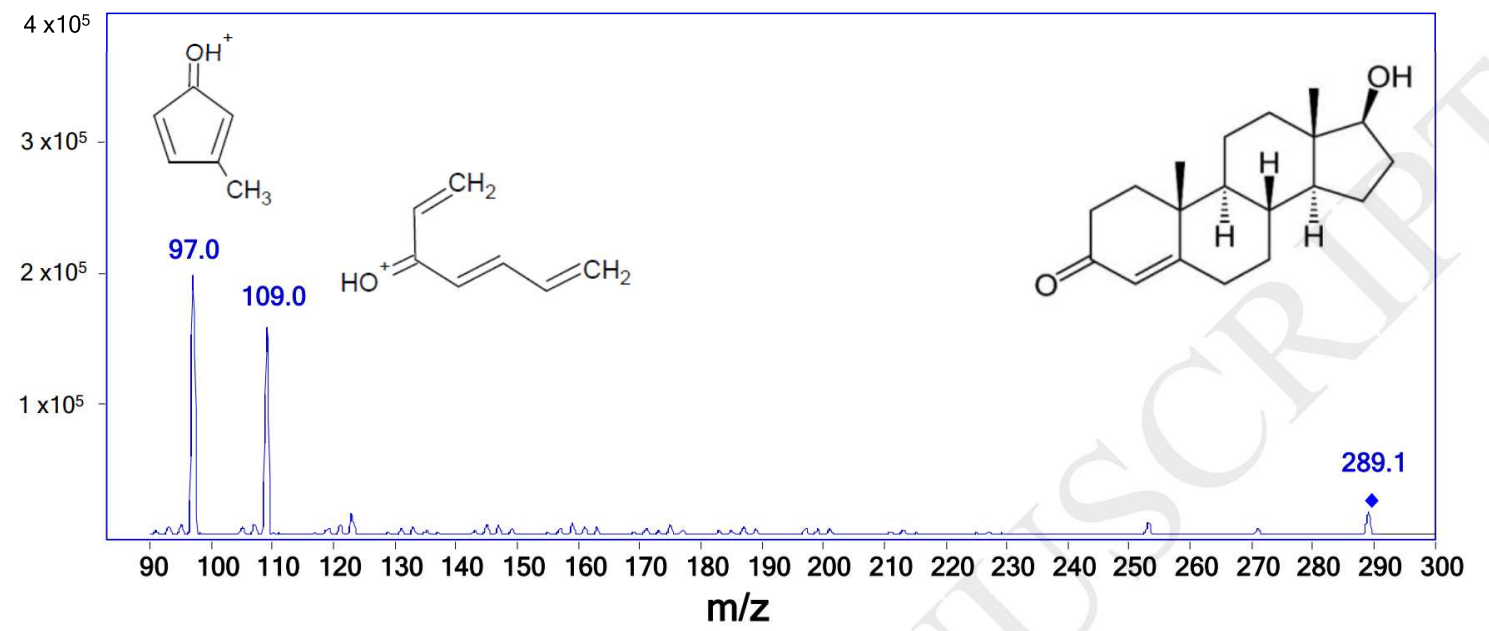

Figure 2. Precursor ion scan from $\mathrm{m} / \mathrm{z} 278$ to 296 of a mixture of natural abundance and ${ }^{13} \mathrm{C}_{2}$ testosterone for product ions from $\mathrm{m} / \mathrm{z} 97$ to100 at a resolution of $F W H M=7.9$ (A), 9.2 (B) and 11.6 (C) and for product ions from m/z 109 to 112 at a resolution of $F W H M=7.9$ (D), 9.2 (E) and $11.6(\mathrm{~F})$.
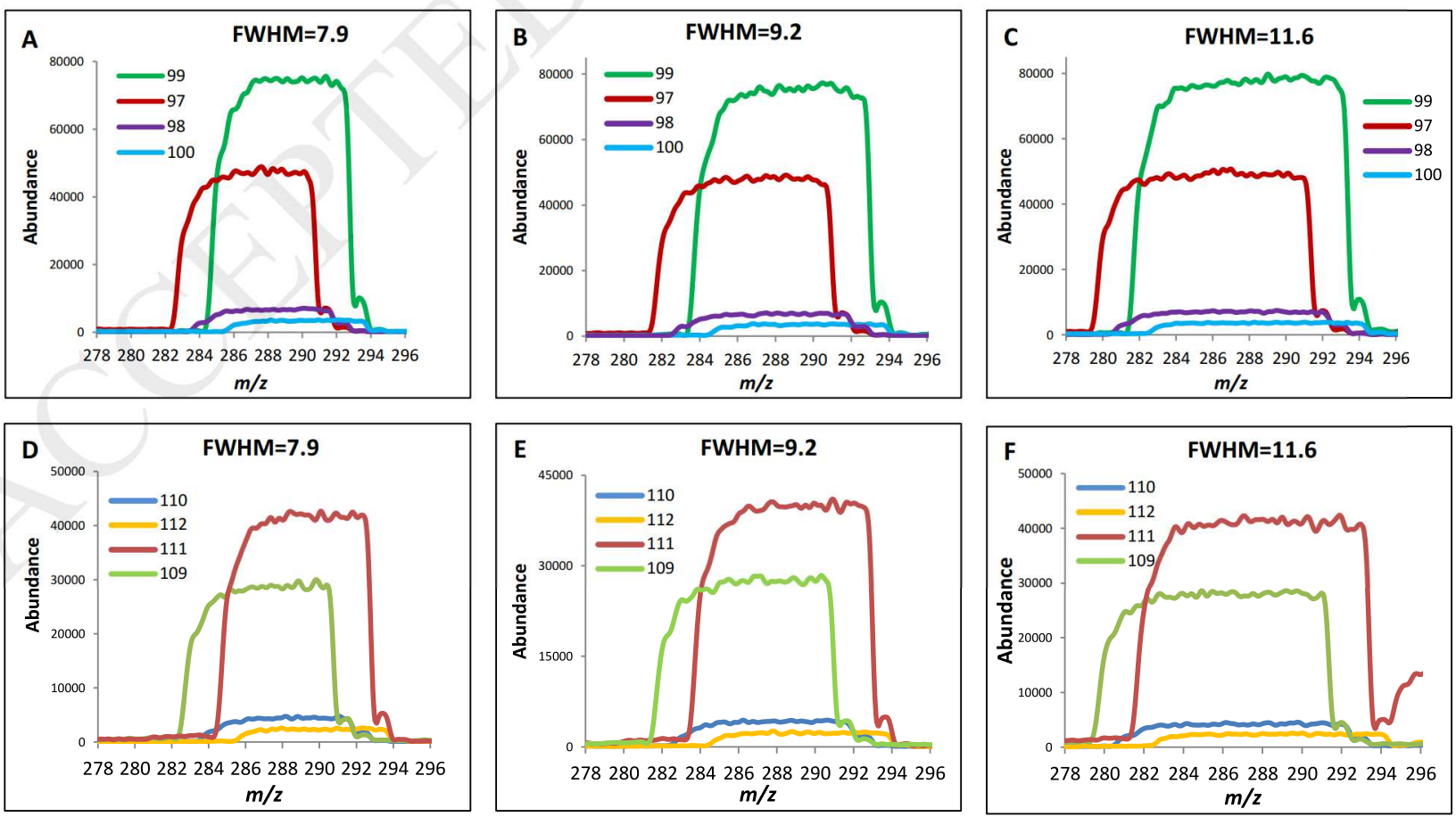
Figure 3. LC-MS/MS chromatogram of the same urine sample of $1.5 \mathrm{ng} \mathrm{g}^{-1}$ analysed in the low resolution SRM mode (blue) and the standard resolution SRM mode (red). The peak for testosterone has a retention time of $7.4 \mathrm{~min}$.

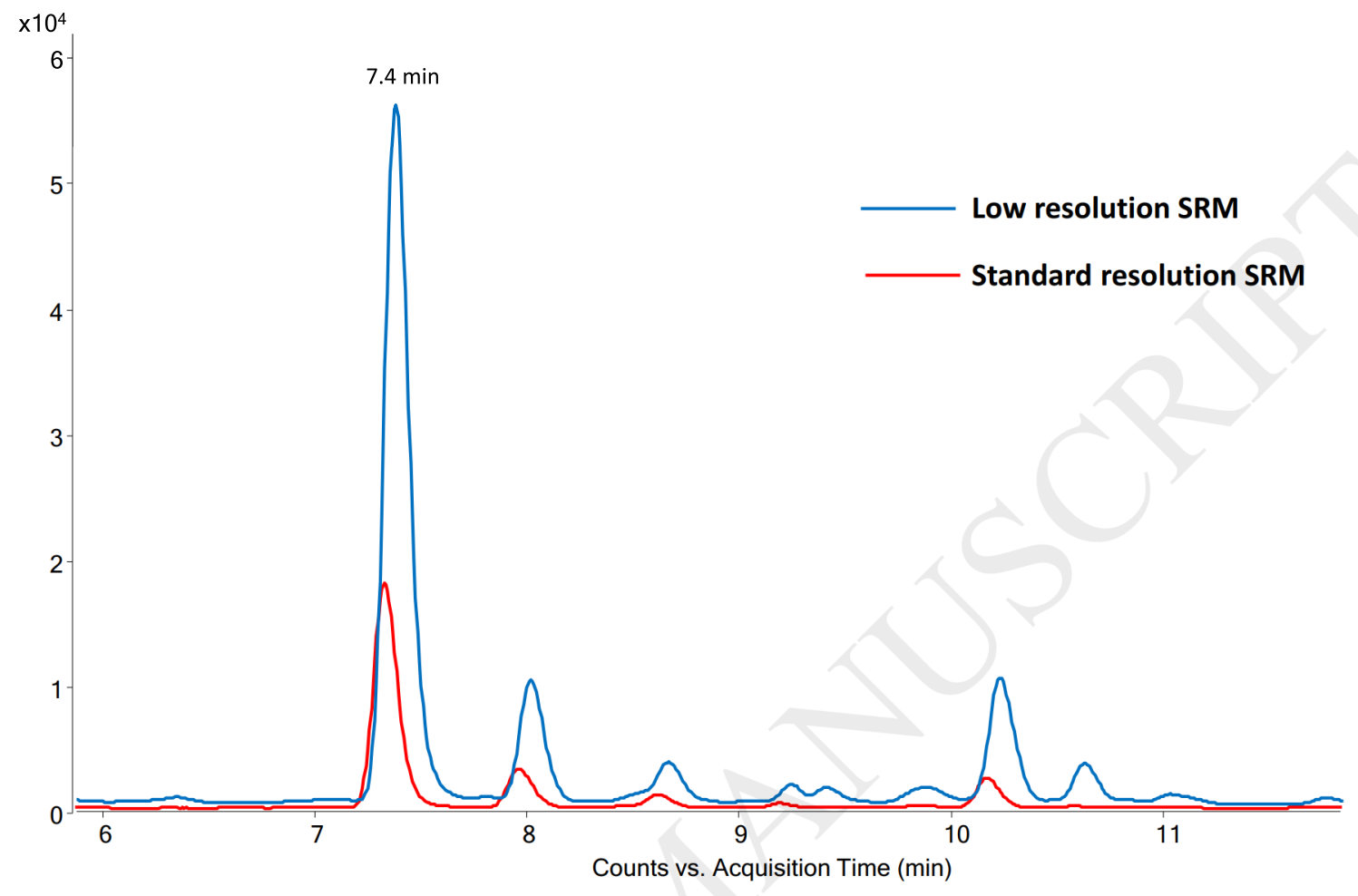




\section{TABLES}

Table 1. Isotopic enrichment (at\%) of the ${ }^{13} \mathrm{C}_{2}$-testosterone employed in this work obtained following the procedure proposed elsewhere [37] in SIM mode and in low resolution SRM (FWHM=11.6).

\begin{tabular}{cccc}
\hline & SIM & Low SRM at $\mathbf{~} / \mathbf{z}=\mathbf{9 9}$ & Low SRM at $\mathbf{~} / \mathbf{z = 1 1 1}$ \\
\hline Tracer solution 1 & 99.21 & 99.21 & 99.12 \\
Tracer solution 2 & 99.22 & 99.43 & 99.26 \\
Tracer solution 3 & 99.22 & 99.46 & 99.27 \\
\hline Average & $99.22 \pm 0.01$ & $99.37 \pm 0.14$ & $99.22 \pm 0.08$ \\
\hline
\end{tabular}

Table 2. Concentration of a ${ }^{13} \mathrm{C}_{2}$-testosterone solution obtained by reverse IDMS in SIM mode and in low resolution SRM at three different FWHM values $(7.9,9.2,11.6)$ for product ions from $\mathrm{m} / \mathrm{z} 97$ to 100 and from $\mathrm{m} / \mathrm{z} 109$ to 112 setting a $\mathrm{m} / \mathrm{z}$ value of 289 in the first quadrupole. The uncertainty values represent the standard deviation of three independent blends.

\begin{tabular}{|c|c|c|c|}
\hline $\begin{array}{l}\text { Acquisition mode } \\
\text { \& Resolution }\end{array}$ & $\begin{array}{c}\mathrm{m} / \mathrm{z}=289.1,290.1 \\
291.1,292.1\end{array}$ & $\begin{array}{l}289 \rightarrow 97,289 \rightarrow 98, \\
289 \rightarrow 99,289 \rightarrow 90\end{array}$ & $\begin{array}{l}289 \rightarrow 109,289 \rightarrow 110, \\
289 \rightarrow 111,289 \rightarrow 112\end{array}$ \\
\hline SIM FWHM=0.7 & $14.07 \pm 0.26$ & & \\
\hline SRM FWHM=7.9 & & $14.71 \pm 0.30$ & $14.34 \pm 0.27$ \\
\hline SRM FWHM=9.2 & & $14.27 \pm 0.25$ & $13.87 \pm 0.27$ \\
\hline SRM FWHM=11.6 & & $14.56 \pm 0.24$ & $14.20 \pm 0.24$ \\
\hline
\end{tabular}

Table 3. Limit of Detection (LOD) and Limit of Quantification (LOQ) calculated from the standard deviation of $n=6$ independent replicates of ultrapure water for the two product ion clusters at $m / z=97$ and $\mathrm{m} / \mathrm{z}=109$.

\begin{tabular}{c|cc}
\hline \multirow{2}{*}{ Replicate } & \multicolumn{2}{|c}{ Blank values $\left(\mathbf{n g ~ g}^{-\mathbf{1}}\right)$} \\
\cline { 2 - 3 } & cluster at $\mathbf{~} / \mathbf{z}=\mathbf{9 7}$ & cluster at $\mathbf{~} / \mathbf{z}=\mathbf{1 0 9}$ \\
\hline 1 & $0.003 \pm 0.003$ & $0.006 \pm 0.008$ \\
2 & $0.009 \pm 0.003$ & $0.008 \pm 0.001$ \\
3 & $0.003 \pm 0.002$ & $0.002 \pm 0.001$ \\
4 & $0.004 \pm 0.003$ & $0.004 \pm 0.004$ \\
5 & $0.006 \pm 0.002$ & $0.005 \pm 0.002$ \\
6 & $0.004 \pm 0.002$ & $0.002 \pm 0.002$ \\
Average & 0.005 & 0.004 \\
Standard deviation (SD) & 0.002 & 0.002 \\
LOD (3SD) & 0.007 & 0.007 \\
LOQ (10SD) & 0.023 & 0.024 \\
\hline
\end{tabular}


Table 4. Recovery values (\%) obtained by the proposed low resolution SRM procedure in ultrapure water fortified with known amounts of natural abundance testosterone at $0.25,0.75,4$ and $7.5 \mathrm{ng} \mathrm{g}^{-1}$ for the two product ion clusters at $\mathrm{m} / \mathrm{z}=97$ and $\mathrm{m} / \mathrm{z}=109$.

\begin{tabular}{|c|c|c|c|c|c|c|}
\hline Sample & $\begin{array}{l}\text { Concentration } \\
\text { added }\left(\text { ng g }^{-1}\right)\end{array}$ & $\begin{array}{c}\text { Theoretical } \\
\text { concentration }\left(\mathrm{ng} \mathrm{g}^{-1}\right)\end{array}$ & $\begin{array}{c}\text { Experimental concentration }\left(\mathrm{ng} \mathrm{g}^{-1}\right) \\
\text { for cluster at } \mathrm{m} / \mathrm{z}=97\end{array}$ & $\begin{array}{l}\text { Recovery (\%) for } \\
\text { cluster at } \mathrm{m} / \mathrm{z}=97\end{array}$ & $\begin{array}{c}\text { Experimental concentration }\left(\mathrm{ng} \mathrm{g}^{-1}\right) \\
\text { for cluster at } \mathrm{m} / \mathrm{z}=109\end{array}$ & $\begin{array}{l}\text { Recovery }(\%) \text { for } \\
\text { cluster at } \mathrm{m} / \mathrm{z}=109\end{array}$ \\
\hline 1.1 & & 0.248 & $0.218 \pm 0.004$ & $88.0 \pm 1.8$ & $0.235 \pm 0.011$ & $102.7 \pm 4.8$ \\
\hline 1.2 & 0.25 & 0.237 & $0.213 \pm 0.001$ & $90.0 \pm 0.6$ & $0.224 \pm 0.007$ & $102.1 \pm 3.3$ \\
\hline 1.3 & & 0.256 & $0.223 \pm 0.003$ & $86.9 \pm 1.2$ & $0.243 \pm 0.001$ & $102.8 \pm 0.5$ \\
\hline Average recovery & & & & $88.3 \pm 1.7$ & & $102.5 \pm 2.9$ \\
\hline 2.1 & 075 & 0.745 & $0.767 \pm 0.002$ & $102.9 \pm 0.3$ & $0.764 \pm 0.005$ & $102.5 \pm 0.7$ \\
\hline 2.2 & 0.10 & 0.750 & $0.757 \pm 0.003$ & $100.9 \pm 0.4$ & $0.768 \pm 0.013$ & $102.4 \pm 1.7$ \\
\hline Average recovery & & & & $101.4 \pm 1.1$ & & $102.4 \pm 1.2$ \\
\hline 3.1 & & 4.018 & $3.962 \pm 0.036$ & $98.6 \pm 0.9$ & $3.944 \pm 0.014$ & $98.2 \pm 0.3$ \\
\hline 3.2 & 4 & 4.130 & $4.080 \pm 0.004$ & $98.8 \pm 0.1$ & $4.056 \pm 0.030$ & $98.2 \pm 0.7$ \\
\hline 3.3 & & 4.021 & $3.962 \pm 0.015$ & $98.5 \pm 0.4$ & $3.963 \pm 0.010$ & $98.5 \pm 0.2$ \\
\hline Average recovery & & & & $98.6 \pm 0.5$ & & $98.3 \pm 0.5$ \\
\hline 4.1 & & 7.422 & $7.324 \pm 0.015$ & $98.7 \pm 0.2$ & $7.316 \pm 0.028$ & $98.6 \pm 0.4$ \\
\hline 4.2 & 7.5 & 7.480 & $7.375 \pm 0.016$ & $98.6 \pm 0.2$ & $7.355 \pm 0.008$ & $98.3 \pm 0.1$ \\
\hline 4.3 & & 7.578 & $7.456 \pm 0.004$ & $98.4 \pm 0.1$ & $7.457 \pm 0.043$ & $98.4 \pm 0.6$ \\
\hline Average recovery & & & & $98.6 \pm 0.6$ & & $98.4 \pm 0.4$ \\
\hline
\end{tabular}


Table 5. Recovery values (\%) obtained by the proposed low resolution SRM procedure in a urine sample fortified with known amounts of natural abundance testosterone at $0.25,0.75,4$ and $7.5 \mathrm{ng} \mathrm{g}^{-1}$ for the two product ion clusters at $\mathrm{m} / \mathrm{z}=97$ and $\mathrm{m} / \mathrm{z}=109$.

\begin{tabular}{|c|c|c|c|c|c|c|}
\hline Sample & $\begin{array}{l}\text { Concentration } \\
\text { added }\left(\mathrm{ng} \mathrm{g}^{-1}\right)\end{array}$ & $\begin{array}{c}\text { Theoretical } \\
\text { concentration }\left(\mathrm{ng} \mathrm{g}^{-1}\right)\end{array}$ & $\begin{array}{c}\text { Experimental concentration }\left(\mathrm{ng} \mathrm{g}^{-1}\right) \\
\text { for cluster at } \mathrm{m} / \mathrm{z}=97\end{array}$ & $\begin{array}{l}\text { Recovery (\%) for } \\
\text { cluster at } \mathrm{m} / \mathrm{z}=97\end{array}$ & $\begin{array}{c}\text { Experimental concentration }\left(\mathrm{ng} \mathrm{g}^{-1}\right) \\
\text { for cluster at } \mathrm{m} / \mathrm{z}=109\end{array}$ & $\begin{array}{l}\text { Recovery (\%) for } \\
\text { cluster at } \mathrm{m} / \mathrm{z}=109\end{array}$ \\
\hline 1.1 & & 1.249 & $1.354 \pm 0.023$ & $108.5 \pm 1.9$ & $1.215 \pm 0.038$ & $97.3 \pm 3.0$ \\
\hline 1.2 & 0.25 & 1.239 & $1.279 \pm 0.014$ & $103.2 \pm 1.2$ & $1.185 \pm 0.028$ & $95.6 \pm 2.3$ \\
\hline 1.3 & & 1.250 & $1.373 \pm 0.046$ & $109.8 \pm 3.7$ & $1.244 \pm 0.028$ & $99.6 \pm 2.2$ \\
\hline Average recovery & & 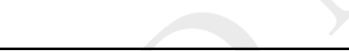 & & $107.2 \pm 3.7$ & & $97.5 \pm 2.8$ \\
\hline 2.1 & & 1.695 & $1.852 \pm 0.027$ & $109.2 \pm 1.6$ & $1.833 \pm 0.036$ & $108.2 \pm 2.1$ \\
\hline 2.2 & 0.75 & 1.690 & $1.777 \pm 0.017$ & $105.2 \pm 1.0$ & $1.749 \pm 0.006$ & $103.5 \pm 0.4$ \\
\hline 2.3 & & 1.694 & $1.792 \pm 0.016$ & $105.8 \pm 0.9$ & $1.756 \pm 0.001$ & $103.7 \pm 0.1$ \\
\hline Average recovery & & & & $106.7 \pm 2.2$ & & $105.1 \pm 2.5$ \\
\hline 3.1 & & 4.720 & $4.789 \pm 0.028$ & $101.5 \pm 0.6$ & $4.842 \pm 0.003$ & $102.6 \pm 0.1$ \\
\hline 3.2 & & 4.733 & $4.836 \pm 0.057$ & $102.2 \pm 1.2$ & $4.838 \pm 0.024$ & $102.2 \pm 0.5$ \\
\hline 3.3 & & 4.712 & $4.846 \pm 0.020$ & $102.8 \pm 0.4$ & $4.838 \pm 0.014$ & $102.7 \pm 0.3$ \\
\hline Average recovery & & & \pm & $102.2 \pm 0.9$ & & $102.5 \pm 0.4$ \\
\hline 4.1 & & 7.933 & $8.069 \pm 0.018$ & $101.7 \pm 0.2$ & $8.159 \pm 0.009$ & $102.9 \pm 0.1$ \\
\hline 4.2 & 7.5 & 8.554 & $8.680 \pm 0.030$ & $101.5 \pm 0.4$ & $8.800 \pm 0.049$ & $102.9 \pm 0.6$ \\
\hline 4.3 & & 8.197 & $8.286 \pm 0.034$ & $101.1 \pm 0.4$ & $8.428 \pm 0.034$ & $102.8 \pm 0.4$ \\
\hline Average recovery & & & & $101.4 \pm 0.4$ & & $102.8 \pm 0.4$ \\
\hline
\end{tabular}


Table 6. Testosterone concentrations ( $\mathrm{ng} \mathrm{g}^{-1}$ ) obtained in 2 male urine samples (M1 and M2) and 3 female urine samples (F1, F2 and F3) analysed by isotope dilution mass spectrometry using three different methods: 1) multiple linear regression combined with Low resolution SRM, 2) multiple linear regression combined with standard resolution SRM and 3) classical IDMS using a calibration graph. Uncertainty values correspond to the standard deviation of 3 independent injections in the LC-MS/MS system. Values in brackets correspond to the relative standard deviation (\%).

\begin{tabular}{|c|c|c|c|}
\hline Method & Low resolution SRM & Standard Resolution SRM & Calibration Graph \\
\hline $\begin{array}{c}\text { SRM } \\
\text { transitions }\end{array}$ & $\begin{array}{l}289.1 \rightarrow 97.1,289.1 \rightarrow 98.1 \\
289.1 \rightarrow 99.1,289.1 \rightarrow 100.1\end{array}$ & $\begin{array}{l}289.1 \rightarrow 97.1,290.1 \rightarrow 98.1 \\
291.1 \rightarrow 99.1,292.1 \rightarrow 100.1\end{array}$ & $289.1 \rightarrow 97.1,291.1 \rightarrow 99.1$ \\
\hline M1 & $52.2 \pm 0.2(0.5)$ & $54.1 \pm 0.6(1.1)$ & $52.3 \pm 0.6(1.1)$ \\
\hline M2 & $13.14 \pm 0.03(0.2)$ & $13.92 \pm 0.05(0.4)$ & $13.69 \pm 0.05(0.4)$ \\
\hline F1 & $1.570 \pm 0.007(0.4)$ & $1.81 \pm 0.01(0.7)$ & $1.81 \pm 0.01(0.7)$ \\
\hline F2 & $15.67 \pm 0.09(0.6)$ & $16.8 \pm 0.1(0.7)$ & $16.3 \pm 0.1(0.6)$ \\
\hline F3 & $1.14 \pm 0.03(2.6)$ & $1.305 \pm 0.001(0.1)$ & $1.320 \pm 0.002(0.1)$ \\
\hline $\begin{array}{c}\text { SRM } \\
\text { transitions }\end{array}$ & $\begin{array}{l}289.1 \rightarrow 109.1,289.1 \rightarrow 110.1, \\
289.1 \rightarrow 111.1,289.1 \rightarrow 112.1\end{array}$ & $\begin{array}{l}289.1 \rightarrow 109.1,290.1 \rightarrow 110.1, \\
291.1 \rightarrow 111.1,292.1 \rightarrow 111.1\end{array}$ & $\begin{array}{l}289.1 \rightarrow 109.1 \\
291.1 \rightarrow 111.1\end{array}$ \\
\hline M1 & $52.98 \pm 0.08(0.2)$ & $55.00 \pm 0.07(0.1)$ & $53.12 \pm 0.07(0.1)$ \\
\hline M2 & $13.56 \pm 0.01(0.1)$ & $14.07 \pm 0.03(0.2)$ & $13.75 \pm 0.03(0.2)$ \\
\hline F1 & $1.85 \pm 0.03(1.9)$ & $1.83 \pm 0.02(1.3)$ & $1.7 \pm 0.1(8.5)$ \\
\hline F2 & $16.8 \pm 0.1(0.6)$ & $16.8 \pm 0.4(2.1)$ & $16.3 \pm 0.4(2.1)$ \\
\hline F3 & $1.51 \pm 0.03(1.8)$ & $1.33 \pm 0.01(0.9)$ & $1.30 \pm 0.01(0.9)$ \\
\hline
\end{tabular}

Table 7. Results obtained in the analysis of certified reference material NMIA MX005 (freeze-dried human urine) using the proposed low resolution SRM method. Uncertainty values of the replicates correspond to the 1s standard deviation of three independent injections in the LC-MS/MS system. Uncertainty of the average values corresponds to the standard deviation of the nine replicates.

\begin{tabular}{|c|c|c|c|c|}
\hline & \multicolumn{2}{|c|}{ Cluster at $\mathrm{m} / \mathrm{z}=97+$} & \multicolumn{2}{|c|}{ Cluster at $\mathrm{m} / \mathrm{z}=109$} \\
\hline & Average (ng g ${ }^{-1}$ ) & Uncertainty & Average $\left(\mathrm{ng} \mathrm{g}^{-1}\right)$ & Uncertainty \\
\hline \multirow{3}{*}{ Day 1} & 38.8 & 0.2 & 39.2 & 0.2 \\
\hline & 39.0 & 0.2 & 39.5 & 0.1 \\
\hline & 40.1 & 0.1 & 41.0 & 0.2 \\
\hline \multirow{3}{*}{ Day 2} & 38.8 & 0.2 & 39.3 & 0.1 \\
\hline & 38.6 & 0.7 & 38.7 & 0.8 \\
\hline & 38.6 & 0.3 & 38.9 & 0.2 \\
\hline \multirow{3}{*}{ Day 3} & 39.3 & 0.1 & 39.5 & 0.2 \\
\hline & 39.4 & 0.1 & 39.6 & 0.1 \\
\hline & 39.1 & 0.1 & 39.6 & 0.3 \\
\hline Average & \multicolumn{2}{|c|}{39.1} & \multicolumn{2}{|c|}{39.5} \\
\hline Uncertainty & \multicolumn{2}{|c|}{0.5} & \multicolumn{2}{|c|}{0.7} \\
\hline$\%$ RSD & \multicolumn{2}{|c|}{1.2} & \multicolumn{2}{|c|}{1.7} \\
\hline \multicolumn{2}{|c|}{ Certified value } & \multicolumn{2}{|c|}{$39.9 \pm 1.7$} & \\
\hline
\end{tabular}

